or if her social circumstances/medical comorbidities change?

There are also other advantages in sending 'unsolicited mail' to our patients that Dr Greenhalgh overlooks. For example, such offers of screening, even if they are declined, can serve to remind infrequent attenders of the existence of their GP and encourage attendance for other problems.

Dr Greenhalgh refers to a few of her patients who have negative attitudes towards proactive care' and one patient in particular who felt that her biometric data in the absence of symptoms are none of anyone's business'. Surely it is our role as primary care clinicians to challenge attitudes of this sort and, so far as it is reasonable, encourage all patients to participate willingly and actively in taking responsibility for their long-term health, rather than allowing a select few to opt out of health checks on a default basis.

\section{Zain Bamber,}

GP Locum, Harrow, London.

E-mail: zapbazladoctors.org.uk

\section{REFERENCE}

1. Greenhalgh T. Outside the Box: Proactive care the patients' right to choose. Br J Gen Pract 2013; 63(606): 37 .

\section{Symptoms and risk factors to identify people with suspected cancer in primary care}

Julia Hippisley-Cox and her team have made great progress in their continuing process of helping GPs estimate a patient's risk of cancer.1,2 I do suggest caution in the use of their current QCancer ${ }^{\circledR}$ risk calculators ${ }^{3,4}$ for three reasons though.

First, the risk calculators don't as yet include important data such as the doctor's clinical examination findings or recent negative results of screening by mammography, cervical smears, and colonoscopy.

Second, predictive values of symptoms, signs, and test results (indicants) depend on the context in which the data were collected. ${ }^{5}$ Predictive values for indicants in the QCancer risk calculators were derived and validated using coded data recorded during general practice consultations These predictive values therefore don't necessarily apply to data recorded by patients themselves or by doctors on checklists, nor to uncoded indicants. This isn't made clear to members of the public visiting the QCancer website who are told: 'You can use this calculator to work out your risk of having a cancer as yet undiagnosed by answering some simple questions. ${ }^{3,4}$ Nor is it made clear to doctors who are told that the template would then help structured data entry of other related symptoms including significant negative findings and the results generated automatically. ${ }^{1,2}$

Third, a digital risk score replacing or accompanying the traditional analogue clinical note will have unpredictable, perhaps undesirable, consequences. Diagnosis and risk assessment are improved by looking at the clinical information available from different angles. These calculators provide an additional perspective. How clear a view they give us is questionable.

\section{Wilfrid Treasure,}

Whalsay Health Centre, Symbister, Whalsay, Shetland, ZE2 9AE.

E-mail: wtreasureagmail.com

\section{REFERENCES}

1. Hippisley-Cox J, Coupland C. Symptoms and risk factors to identify men with suspected cancer in primary care: derivation and validation of an algorithm. Br J Gen Pract 2013; DOI: 10.3399/ bjgp13X660724.

2. Hippisley-Cox J, Coupland C. Symptoms and risk factors to identify women with suspected cancer in primary care: derivation and validation of an algorithm. Br J Gen Pract 2013; DOI: 10.3399/ bjgp13X660733.

3. QCancer-2013-male. http://www.qcancer.org/ male/index.php laccessed 5 Feb 2013).

4. QCancer-2013-female. http://www.qcancer.org/ female/index.php (accessed 5 Feb 2013).

5. Treasure W. Diagnosis and risk management in primary care: words that count, numbers that speak. Milton Keynes: Radcliffe Publishing, 2011.

DOI: 10.3399/bjgp13X664126

\section{Authors' response}

Thank you for the opportunity to respond to Dr Wilfred Treasure's letter ${ }^{1}$ regarding our two papers. ${ }^{2,3}$ The QCancer $^{\circledR}$ algorithms are an evolving set of risk algorithms to quantify the risk of a previously undetected cancer. They are intended to support clinical decision making and we are currently carrying out a pilot to inform how best to implement them. The algorithms currently combine multiple risk factors and symptoms to give a global risk of cancer and risks of individual cancer types. Dr Treasure is correct in saying that the QCancer algorithms do not currently include results of clinical examinations; it's possible these could be added in a future version of the tool should the relevant data be coded in the clinical record. However, as we say in the paper, QCancer is intended for use in a primary healthcare setting to help doctors assess which patients to send for investigations such as colonoscopy. Therefore the tool does not include the results of colonoscopy or other diagnostic tests as that is not the purpose of the tool.

In response to Dr Treasure's second point we agree that the predictive values are based on data recorded during general practice consultations. The information on the QCancer website states the following 'This website is primarily intended for doctors and nurses working in general practice and for academics who are interest in the underlying research. Patients are welcome to read this information and use the calculator together with their doctor so that any symptoms or concerns can be addressed within a healthcare setting. All medical decisions need to be taken by a patient in consultation with their doctor. QCancer works out the risk of a patient having a current but as yet undiagnosed cancer-taking account of their risk factors and current symptoms. It does not give a diagnosis of cancer, but a risk.

The paper offers possibilities on how QCancer can be implemented into clinical settings as Dr Treasure points out. The paper is not a detailed guide on the implementation but does offer several possibilities including the use of structured templates. Such templates are already commonly used to assist in data entry across a wide range of clinical areas including risk-assessment tools and their use is likely to improve the ascertainment and recording of positive and negative symptoms over time. This will enhance both the medical record in its primary purpose but also future updates of the QCancer tool.

Julia Hippisley-Cox,

University of Nottingham, Division of Primary Care, 13th Floor, Tower Building, Nottingham, NG2 7RD.

E-mail: juliahippisleycoxagmail.com

Carol Coupland, 